\title{
Weather and the Earth's rotation
}

A model calculation has shown how the speed of the Earth's rotation differs between the northern summer and the northern winter because of the seasonal variation of the force between the atmosphere and the spinning Earth.

EVERYBODY knows that the speed of the Earth's rotation is not as strictly constant as those who sought to use the Earth as an accurate timekeeper were fond of supposing, but the causes of the measured fluctuations in the length of the day, or LOD, remain to be unravelled. That there should be a secular trend to less rapid rotation on account of tidal interactions within the Earth-Sun-Moon system is of course well understood, and is the chief reason for the now routine annual retrospective corrections, of the order of a few milliseconds, of the length of previous years. There are also regular seasonal variations of the speed of rotation occasioned by the different responses of the two hemispheres to solar heating.

But the short-term fluctuations of rotation rate are much less well understood. The notion that variations of weather patterns that change the angular momentum of the Earth's atmosphere may be responsible is natural enough, but there has been no compelling demonstration that the effects of weather are sufficient to account for the observed fluctuations of rotation rate. One obvious difficulty is that direct correlation of fluctuations of the LOD with measurements of the angular momentum of the atmosphere would require an unattainable degree of sophistication in meteorological measurement. But G. J. Boer from the Canadian Climatic Centre at Downsview, Ontario, may now have found a way of settling the issue (Journal of Geophysical Research 95, $5511 ; 1990)$.

The inevitable starting point is the elementary consideration that the angular momentum of the Earth must be constant at least within the framework of the small secular trend to increasing LOD. This in turn implies that the sum of the angular momentum of the solid Earth and the angular momenta of its movable components must be constant. But the movable components are the atmosphere and the oceans. The Earth's molten core is also capable of a fluctuating angular momentum, and indeed does influence the LOD (see J. Lahr, Nature 345, 476; 1990) but on much longer timescales than are considered here.

Boer's trick is to use the Canadian Climatic Centre's model of the general atmospheric circulation to calculate the transfer of angular momentum between the different components of the spinning
Earth. Of necessity, this approach cannot yield a direct correlation between particular weather patterns and particular excursions of the Earth's rotation rate, but Boer is at least able to demonstrate that transfers of angular momentum between the atmosphere and the solid Earth can indeed account for LOD fluctuations of the magnitude observed. Boer acknowledges that his work is by no means the first of its kind and in particular refers to a study five years ago by R. Swinbank at the UK Meteorological Office.

The interaction between the atmosphere and the solid Earth consists of the stresses caused at the interface between them by relative movement of the atmosphere otherwise known as wind. For practical purposes, three parts of the Earth's surface require separate treatment.

First, mountain ridges and other topographical variations of surface altitude present obstacles to wind; the stresses they cause are most simply determined by the differences of pressure across them. But there are also frictional forces at topographically unremarkable solid surfaces, while at oceanic parts of the interface there may in principle be a literal transfer of angular momentum from the atmosphere to the oceans rather than a stress. Plainly it is a matter of some importance to estimate the relative magnitude of these different contributions to the stress on the spinning Earth and thus to the torque which affects its rotation rate. Boer does acknowledge that Swinbank's earlier work had shown mountain ranges to be dominant.

The procedure is straightforward. In the style of atmospheric modellers everywhere, Boer uses a set of experimental measured data of the condition of the atmosphere as initial conditions for his model, discarding predictions during an initial period while the model settles down. In practice, he then allowed his model to run for the equivalent of 20 years and used the stored data produced as a basis for data calculations. As atmospheric models go, the Canadian is reasonably sophisticated. It allows for radiative processes within its ten-level vertical structure, but sea surface temperatures and average cloudiness must be provided empirically. The model does not accommodate phenomena such as the $\mathrm{El}$ Niño mechanism, which may nevertheless be highly relevant for the issue of the Earth's angular momentum.

For outsiders, the sheer size of the quantities involved, however unsurprising, is bound to be striking. In SI units ( $\mathrm{kg}$ $\mathrm{m}^{2} \mathrm{~s}^{-1}$ for angular momentum), the angular momentum of the atmosphere appears to fluctuate with the seasons between 15.0 and 25.0 times $10^{25} \mathrm{~kg} \mathrm{~m}^{2} \mathrm{~s}^{-1}$. The atmosphere in other words is a gigantic flywheel of sufficient size to affect the spinning of the solid Earth over which it lies. As it happens, the angular momentum of the atmosphere as a whole is greatest in the northern spring (March and April) and least in the northern summer (June and July). That there should be such an asymmetry is not surprising. The angular momentum of the atmosphere is necessarily a balance between the easterly winds of the tropics and the westerlies that prevail in mid-latitudes. That these balance differently in the two hemispheres is apparent from Boer's calculations of the angular momenta in the separate hemispheres; that in the Northern Hemisphere falls to zero in the corresponding summer.

The calculations also show the relative importance of the various forces on the spinning Earth which lead to the observed fluctuations of the LOD. Generally speaking, the torque on the spinning Earth transmitted to the oceanic surface is small compared to the forces transmitted through land surfaces, at mountains and elsewhere. Moreover, the oceanic forces are fairly constant from one season to another. Indeed, such variations of the oceanic forces as there are appear most conspicuously at mid-latitudes in the Southern Hemisphere.

But Boer cautiously acknowledges that the calculations of the torque transmitted through the interface between the atmosphere and the spinning Earth are still incomplete. In one particular respect, for example, it appears to be accepted that the sensitivity of the model calculations to the assumptions made about the topography of the Earth is too great for comfort. Even so, to have made such a detailed compilation of the forces that may influence the spinning rate and thus the length of day is a considerable achievement with which timekeepers, not to mention meteorologists, will have much fun.

John Maddox 